Cahiers $d u$ MONDE RUSSE

\section{Cahiers du monde russe}

Russie - Empire russe - Union soviétique et États indépendants

$50 / 4 \mid 2009$

Varia

\title{
Catherine Depretto, Le Formalisme en Russie
}

\section{Leonid Heller}

\section{OpenEdition \\ Journals}

Édition électronique

URL : https://journals.openedition.org/monderusse/7243

DOI : $10.4000 /$ monderusse. 7243

ISSN : $1777-5388$

Éditeur

Éditions de l'EHESS

Édition imprimée

Date de publication : 15 décembre 2009

Pagination : $924-926$

ISBN : 978-2-7132-2261-0

ISSN : $1252-6576$

\section{Référence électronique}

Leonid Heller, «Catherine Depretto, Le Formalisme en Russie », Cahiers du monde russe [En ligne], 50/4 | 2009, mis en ligne le 12 janvier 2011, consulté le 02 septembre 2022. URL : http://

journals.openedition.org/monderusse/7243; DOI : https://doi.org/10.4000/monderusse.7243

Ce document a été généré automatiquement le 2 septembre 2022.

Tous droits réservés 


\title{
Catherine Depretto, Le Formalisme en Russie
}

\author{
Leonid Heller
}

\section{RÉFÉRENCE}

Catherine DEPRETTO, Le Formalisme en Russie. Paris : Institut d'Études slaves, 2009, $331 \mathrm{p}$.

1 C'est une excellente idée qu'a eue Catherine Depretto lorsqu'elle décida de réunir dans un volume ses articles sur les formalistes russes. Son livre, qui témoigne de la fascination que continue d'exercer une pensée née il y a bientôt cent ans, apporte un nouveau souffle à un domaine de recherche où tout semble avoir déjà été dit après les mises au point de Michel Aucouturier, de Marc Weinstein, après la livraison "formaliste " de la revue Europe. À observer ce domaine d'ailleurs, on se rend compte que la santé des études russes en France est moins mauvaise qu'on le dit parfois. Composé d'une vingtaine de textes publiés entre 1977 et 2009, l'ouvrage recensé n'a rien d'un patchwork ; au contraire, il parvient à une remarquable unité. En se démarquant d'habituelles synthèses théoriques et en se tournant vers les personnes et les circonstances qui firent naître et évoluer le formalisme, Catherine Depretto se fonde sur la connaissance des archives et des documents récemment rendus publics; sa factographie est à l'échelle humaine et personnelle. De là vient la cohérence de l'ensemble.

2 Le livre est introduit par un imposant panorama de la réception actuelle des formalistes, tant à l'intérieur qu'à l'extérieur de la Russie. Ensuite, bousculant la chronologie de leur publication, les textes se répartissent en quatre sections pour proposer un cheminement progressif - qui correspond assez bien à la chronologie historique - à travers la problématique et les destinées complexes du mouvement. On rencontre d'abord les "Précurseurs»: les symbolistes, bien entendu, Andrej Belyj en tête, mais aussi des jeunes universitaires qui font figure de " préformalistes ». Inspirés par leurs maîtres, par les idées venues de l'Occident (en premier lieu, de l'Allemagne), par l'expérimentation 
symboliste, ils s'activent à l'université de Saint-Pétersbourg, dans la faculté des études romano-germaniques, dans le séminaire pouchkiniste de Vengerov, dans des cercles affiliés. Des futurs membres d'OPOJAZ sont là, mais aussi d'autres dont les noms sont peu ou pas connus, tels Nikolaj Nedobrovo ou Mihail Lopatto, et ceux que l'on connaît, tel Julian Oksman, sans les relier au même contexte. La deuxième partie « Le Formalisme et son histoire »- réserve une place importante, à côté du groupe pétersbourgeois d'OPOJAZ que l'on privilégie systématiquement parmi les branches de l'arborescence formaliste, au Cercle linguistique de Moscou, aux échanges entre deux centres, à leur convergences et divergences, à la relève - compagnons de route, disciples -, à des épisodes peu connus, comme celui de 1928, lorsque Jakobson et l'OPOJAZ tentent de relancer le projet formaliste. La troisième partie, intitulée " Formalistes et poètes ", confronte la conception de la parole poétique et, plus particulièrement, celle de la poésie lyrique développée par Tynjanov, à l'impact direct sur le formalisme, au travers de contacts personnels et/ou de leur création, de poètes tels que Majakovskij, Esenin, Mandel'štam, Pasternak. La partie finale, « Prolongements », regroupe des variations sur l'histoire du formalisme: le destin d'Evgenij Polivanov, le roman à clé de Veniamin Kaverin, Tynjanov prosateur enfin, dont la situation au sein de la culture soviétique peut se lire en filigrane dans ses récits de visiteurs étrangers en Russie et de Russes visitant l'étranger.

Plus qu'un simple regroupement thématique, cette partition de l'ouvrage rend apparent le véritable objectif de celui-ci, au-delà de sa préoccupation factographique. Il s'agit d'une remise en question de certaines lectures trop répandues du phénomène formaliste. Plusieurs thèses se dégagent, couplées aux sujets évoqués. L'émergence du formalisme, on l'a vu, n'apparaît plus comme un miracle provoqué uniquement par le chambardement futuriste. Elle est rendue possible par l'activité bouillonnante de tout un milieu philologique engagé dans un échange serré avec le milieu poétique - intrication que Catherine Depretto voit comme un trait spécifique du modernisme russe. Conséquence : l'attitude formaliste peut être liée à d'autres que les futuristes ; aussi ses points de rencontre avec l'acméisme sont-ils repérés, et surtout une étonnante convergence de pensée entre Tynjanov et Mandel'štam. Une autre thèse découle de l'analyse du rapprochement tenté par Jakobson et l'OPOJAZ en 1928 ; elle oppose un refus au diagnostic d'une maladie interne comme cause réelle de la mort du formalisme. "Ainsi a-t-on eu tendance à minimiser la part des interdits politiques pour mettre en avant ses limites méthodologiques, donnant par là-même du mouvement russe l'image d'un précurseur immature face à un structuralisme performant. [...] S'il y a eu un passage de l'un à l'autre, c'est en termes de relève plus que de dépassement théorique qu'il faut l'envisager » (p.144-145). Cette thèse permet de voir d'un œil nouveau l'« évolution postformaliste». D'une part - les contributions plus ou moins récentes d'un Alexandre Galuchkine ou d'une Valérie Pozner vont elles aussi dans ce sens -, les travaux des formalistes dans les années 1930 ne devraient pas être vus sous l'aspect unique d'un abandon de leurs principes de départ. En ce qui me concerne, je renchérirais en suggérant que cet abandon est souvent factice, même dans les écrits tardifs d'un Šklovskij; et que cette production tardive reste d'une lecture enrichissante tant pour un historien que pour un théoricien. D'autre part - et c'est encore moi qui tire cette leçon -, après avoir étudié en détail les conceptions des fondateurs du formalisme, on est désormais autorisé à interroger sa descendance, la cohérence de son héritage et son dépassement dans les travaux de la " jeune génération » (celle de Lidija Čukovskaja ou de Boris Bukštab), 
l'apport particulier de cette dernière, ses autres ramifications possibles, bref, à poser sérieusement la vaste question de l'actualité du formalisme dans sa version russe.

Car j'adhère à l'opinion de Catherine Depretto que les contradictions internes du mouvement n'étaient pas de nature à l'arrêter, ainsi qu'à l'idée d'une grande puissance - au sens de potentiel de modélisation et d'explication - que contient encore la théorie formaliste. J'irais jusqu'à dire qu'il serait moins intéressant d'examiner ce que cette théorie a déjà dit que ce qu'elle peut encore dire aujourd'hui et demain. Un autre moment fort du livre : l'exploration de tout le paysage culturel sur lequel se profilent les tenants du formalisme et qui abrite leur réseau d'interrelations.

5 Certains autres postulats en revanche devraient à mon sens être nuancés. Ainsi, l'affinité entre les positions acméistes et formalistes, parfois bien motivée, semble ailleurs quelque peu forcée. Je continue à croire à une "coupure épistémologique» entre les préoccupations formelles des poètes et philologues des années 1910 - que l'on pourrait comparer à l'étude de symptômes somatiques visant à saisir, selon le principe du parallélisme psychophysique, les états de l'âme - et la démarche formaliste qui prétend battre ce parallélisme en brèche.

6 Il n'existe pas d'ouvrage académique sans défauts. Qu'il me soit permis de pimenter mon compte rendu en en notant quelques-uns. Ainsi, il est dommage que l'excellent portrait de Semën Vengerov soit privé d'une dimension importante : historien et philologue, il a été aussi un des premiers critiques à vouloir saisir toute l'étendue du phénomène moderniste en Russie et à le reconnaître comme un moment important dans l'évolution littéraire. Son anthologie critique Russkaja literatura Xx veka. 1890-1910 (3 vol., 1914-1916), qui faisait autorité en la matière, manque dans la bibliographie du chapitre consacré à son séminaire. Dommage que si peu d'attention soit accordée à Vladimir Propp ou qu'il manque, dans les chapitres sur Tynjanov, la mention d'Arkadij Belinkov. On peut se demander pourquoi, en parlant de Baudouin de Courtenay et de Tadeusz Zielinski, leur origine polonaise n'est pas précisée, alors que le nom de ce dernier s'orthographie à la polonaise et non à la russe. Curieusement, la liste des universités de l'Empire russe (p. 45), pourtant reprise d'un ouvrage historique sérieux, n'inclut pas celle de Varsovie. Or, celleci restait en contact très étroit avec Saint-Pétersbourg. On croirait presque à quelque «intrigue anti-polonaise». L'oubli est certainement anecdotique, mais il empêche l'auteur d'interroger le rôle des savants polonais en tant que relais entre l'occident et la Russie; le postulat sur la spécificité russe des relations intimes entre philologues et poètes s'en trouve affaibli. C'est qu'une tradition semblable est bien ancrée en Pologne (au moins depuis les Philomathes et les Philarètes du temps de Mickiewicz) et en Allemagne où le cercle de Stefan George, le fameux Georgekreis, peut servir de modèle de telles relations.

7 On voit bien que les reproches sont mineurs. Insistons : que l'on soit d'accord ou non avec les thèses mentionnées, il ne sera plus possible de ne pas en tenir compte dans la réflexion sur le formalisme. Mieux, leur audace polémique, l'abondance du matériau bioet factographique, la richesse des notes et de la bibliographie qui gardent la trace virtuellement de toute la littérature secondaire parue sur le sujet du formalisme russe depuis une dizaine d'années - tout cela fait du livre de Catherine Depretto un ouvrage de référence incontournable. 